\title{
SUCCESSION DYNAMICS OF VEGETATION IN THE SLOVAK KARST BIOSPHERE RESERVE LANDSCAPE (WESTERN CARPATHIANS)
}

\author{
IVAN VOLOŠČUK ${ }^{1 *}$, EVA UHLIAROVÁ ${ }^{2}$ PETER SABO ${ }^{1}$, MARTINA ŠKODOVÁ $^{3}$, JURAJ \\ ŠVAJDA ${ }^{2}$
}

${ }^{1}$ Matej Bel University in Banská Bystrica, Institute for Research of landscape and Regions, Cesta na amfiteáter 1,974 00 Banská Bystrica, Slovak Republic; e-mail: ivoloscuk@azet.sk

${ }^{2}$ Faculty of Natural Sciences, Department of Biology and Ecology, Tajovského 41, 97400 Banská Bystrica, Slovak Republic

${ }^{3}$ Faculty of Natural Sciences, Department of Geology and Geography, Tajovského 41, 97400 Banská Bystrica, Slovak Republic

* Author for correspondence

\begin{abstract}
Vološčuk I., Uhliarová E., Sabo P., Škodová M., Švajda J.: Succession dynamics of vegetation in the Slovak Karst Biosphere Reserve Landscape (Western Carpathians). Ekológia (Bratislava), Vol. 35, No. 1, p. 13-31, 2016.

The paper presents results acquired by an ecological analysis of vegetation succession on the field of the Slovak Karst in south-east Slovakia over the past 25 years. The data were collected on permanent research plots etsablished in 1983-1985 on the Plešivská Plateau (part of the Slovak Karst National Park and Biosphere Reserve). Changes in mesic and dry grassland vegetation of permanent field plots were evaluated in the same way both times according to the Central European method. Due to cessation of non-forest vegetationmanagement the majority, permanent plots isgradually overgrownby woody plants. Succession of vegetation runs faster on karst plateaux where the trees and shrubs invasion is accompanied by overgrowing of grassland vegetation with $\mathrm{Bra}$ chypodium pinnatum and Calamagrostis epigejos, which are completly change their species composition. On the steep limestone slopes and karst rocky fields of the lower altitudes the secondary succession is ongoing slower and is subjected mainly to accumulation of soil. Succession of herb layer is firstly reflected in changes of species abundance and later in exchanges of dominant species and plant communities. It was also evaluated succession in two forest habitats. In the community Fagetum typicum was evaluated succession after the artificial spruce wind calamity.
\end{abstract}

Key words: permanent plots, Slovak Karst Biosphere Reserve, grasslands, forest habitats, secondary succession.

\section{Introduction}

Dynamics generally focuses on the changes that are occurring at a certain time. The dynamics of vegetation succession means temporal changes in the vegetation structure (Odum, 
1969; Glenn-Lewin, van der Maarel, 1992). Part of succession on abandoned meadows is the penetration of plant species from the surrounding landscape matrix. Structure of grasslands in the successional process is gradually changing and a longer time horizon meadow plant community was extinguished and habitat occupied the forest vegetation. There is a changecharacteristic landscape. Studying the dynamics of successional and ecological processess is one of the major objectives of ecology (Wu, Hobbs, 2002).

To understand the theory of succession, a hypothesis of thermodynamics theory was proposed (Capra, 1997; Jörgensen, Svirezhev, 2004; Sabo et al., 2011). According to this hypothesis, the driving force behind the development of organisms and ecosystems effort of live system is the most efficient use of available concentrated energy and other natural resources, which is called the maximum power principle. From this perspective, the driving force behind the succession is imbalance between energy input and disbursement (outlay) of the ecosystem. Higher availability of solar energy, for example in gaps (Yamamoto, 2000) compared to the needs of the ecosystem, solves the problem of ecosystem biomass accumulation and creates new dissipative structures and processes, or changes in species composition of biota by creating new, diverse trophic chains that make more efficient use of available energy, which help restore the energy balance of the ecosystem (Kay, 2000; Zhang, Wu, 2002). Living systems are thermodynamically open, which means that they develop and maintain their physical structure, organization and functions depending upon the continuous supply of solar energy.

Consequent accumulation of biomass and nutrients means that more high-quality energy (exergy) is available in the ecosystem, which ,is compelled' to react to this growth of the energy supply and to develop more complex dissipative structures and processes (Jörgensen, Svirezhev, 2004; Sabo et al., 2011). This includes a spontaneous change of the biocoenosis composition in order to increase its capacity to accumulate more exergy (in the form of biomass and nutrients) and to dissipate the incoming solar exergy through more efficient pathways. This process is reflected in the consequent increase of the numbers and proportion of C-strategists and decline of R-strategists and in the increase of the size of the gilds on higher trophic levels, due to richer food supply (i.e. more exergy) available on the lower levels (Würtz, Anilla, 2010).

Plants in the adult stage have developed three types of strategies. Competitors (C) are adapted to environments with low levels of stress and disturbance; stress-tolerators $(S)$ to high stress and low disturbance, and ruderals (R) to low stress and high disturbance (Grime, 2001; Šilc, Čarni, 2005). No plant species have developed a ‘viable strategy` for tackling the combination of high stress and high disturbance. By distinguishing intermediate levels of stress and disturbance, intermediate (so-called secondary) strategy types can be formed: competitive ruderals (C-R) adapted to low stress and moderate disturbance; stress-tolerant ruderals (S-R) adapted to high stress and moderate disturbance; stress-tolerant competitors (S-C) adapted to moderate stress and low disturbance; and C-S-R strategists adapted to moderate stress and moderate disturbance.

Non-equilibrium theory (Pickett et al., 1987; Kay, 2000) predicts that equilibrium cannot be realized because of the interruption of succession towards equilibrium conditions by major disturbances. According to the concept of non-equilibrium thermodynamics, every 
input of energy causes displacement of a living system from the point of thermodynamic equilibrium (Sabo et al., 2011).

The aim of the present paper is to evaluate of vegetation succession in selected stationary areas of the Slovak Karst, which occurred as a result of natural succession and changes in the land use over the last 25 years.

\section{Study area}

The study area is located in Western Carpathians, South-East Slovakia, in the Plešivec Plateau of the Slovak Karst National Park (NP) (Figs 1,2). The area is a series of plateaux whose maximum altitude ranges between 400 and $900 \mathrm{~m}$. The summits of the Plešivec Plateau reach $851 \mathrm{~m}$ (SNC, 2011) and its geographical coordinates are N $48^{\circ} 28^{\prime}-$ $48^{\circ} 43^{\prime}$; E $20^{\circ} 15^{\prime}-21^{\circ} 01^{\prime}$.

The Slovak Karst NP is situated in the northern temperate zone on the junction between oceanic and continental climatic zones. With a mean altitude of $598 \mathrm{~m}$, its climate is transitional between lowland and mountain types, and is modified by the irregularities of the karst relief. Mean annual air temperature ranges from 5.7 to $8.5^{\circ} \mathrm{C}$. The annual average precipitation ranges from 630 to 990 $\mathrm{mm}$, with a growing season average of 400 to 595 mm (SNC, 2011; Rozložník, Karasová, 1994).

The basic geological structural unit of the region is the limestone complexes. The Slovak Karst is a distinct geomorphological unit, classified as a karst with elevated upland plateaux surrounded by steep slopes descending to adjacent basins, valleys and gorges. The plateaux slant from $800-900 \mathrm{~m}$ in the north to $450-500 \mathrm{~m}$ in the south, with a general moderate southwards inclination of 2 to $4^{\circ}$. The originally compact plateau surface has been dissected by stream erosion into subunits. Karst phenomena are created by the corrosive and erosive activities of water in soluble limestones. These are both primary (lapiés, karst depressions and valleys, sinkholes, karst springs, chasms and caves) classified as surface or underground, and secondary (dripstones, travertines).

The variety of soil types (IUSS, 2014) reflects the region's heterogeneous geological composition. Rendzina-type soils occur on carbonate bedrock, with cambisols in adjacent basins; cambisols and luvisols occur on non-carbonate bedrocks and hydromorphic soils on the bottom of valleys. Limestones, dolomites and their scree at the base of slopes are covered by the products of long-term weathering and fossil soils (terrae calcis). In addition, plateau surfaces broken by lapiés and sinkholes are covered by rendzinas, luvisols and protorendzinas (SNC, 2011).

The area of Slovak Karst was officially declared as the Protected Landscape Area (PLA) in 1973 (Rozložník, Karasová, 1994). The territory of PLA was declared a National Park on 1 March 2002. The protected territory has an area of $361.7 \mathrm{~km}^{2}$ and is surrounded by a prevention zone of $383.3 \mathrm{~km}^{2}$.

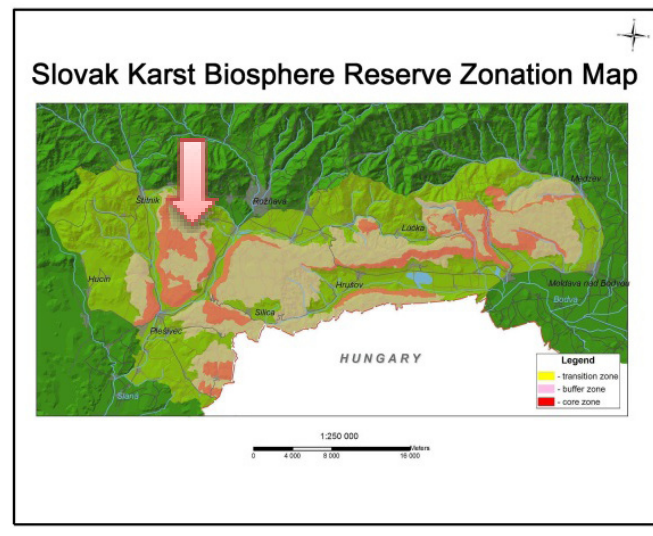

Fig. 1. Position of Plešivská Plateau in Slovak Karts territory.

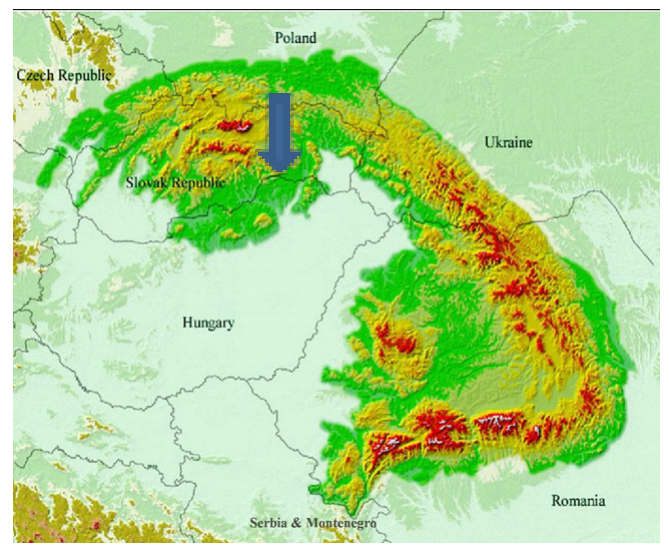

Fig. 2. Situation of Slovak Karst Mts in Carpathians. 
In 1977, the UNESCO designated the PLA and its prevention zone within the international system of biosphere reserves (BR). The caves situated in Slovak Karst National Park and the adjacent Aggtelek National Park in Hungary have been added to the UNESCO World Heritage List on 9 December 1995.

The territory of Slovak Karst NP belongs to the Biogeographical RegionMiddle European Forest (Devillers, Devillers-Terschuren, 1999). Those significant for conservation of biological diversity are European Important Habitats (Anonymous, 1992, Interpretation manual of EU Habitats, 2003).

The landscape of Slovak Karst NP is characterized by the great fragmentation of land property and extensive form of land use. In the last decades, rapid abandonment of mowing and land cultivation has been observed. Woodlands are the predominant biome of the plateaux: of the total area of the NP, forests cover $76 \%$, grasslands and pastures $16 \%$ and arable land $4 \%$. Most of the woodlands are coppice stands derived from repeatedly cut broad-leaved trees, and forest plantations cultivated by foresters. On the plateaux, forestry is predominant, with some agriculture. Settlements and related economic activities are concentrated on the basins and river valleys. The region has an industrial-rural character, and more people are employed in agriculture than industry (SNC, 2011). Forests are a significant landscape element, which create the characteristic feature of the landscape and contribute to its ecological stability (Vološčuk et al., 2011).

\section{Material and methods}

The field work in Plešivská Plateau (Slovak Karst) was conducted during 1983-1985, and 2010-2011. Only a few sporadic investigations were made earlier (Vološčuk, 1993; Vološčuk et al., 2011). The research of forest ecosystems was done in accordance with the Zlatník's geobiocoenological system (Zlatník, 1959), non-forest ecosystems (grasslands) was done in accordance with Zürich- Montpellier methodology (Braun-Blanquet, 1964). The abundance of plant species was estimated using the Braun-Blanquet scale extended by van den Maarel (1979). The results, especially in the area of Plešivská Plateau, were published in several works (Háberová, 1988; Vološčuk, 1988a,b, 1993; Háberová, Karasová, 2002). In addition to the phytosociological registration, trees throughout the area were assessed and measuring the height of trees in selected areas of stationary was done. Measuring the height of trees was carried out on area of $10 \times 20$ $\mathrm{m}$. A comparison of the original and repeated phytocoenological entries were made in the program JUICE (Tichý, 2002; Tichý, Holt, 2006; Tichý, Chytrý, 2006) using the program TWINSPAN (Hill, Šmilauer, 2005). They evaluated the changes in the overall species composition of vegetation, by dominant species and species coverage on the entire stationary surface. For inclusion in the initial and recurrent phytocoenological entries for individual syntaxa, electronic expert system for identification of plant communities was used. The results were corrected according to the latest data on grassland vegetation of Slovakia and Slovak Karst (Janišová et al., 2007b; Dúbravková-Michálková et al., 2008).

Entries were saved in a database program TURBOVEG (Hennekens, Schaminée, 2001). The nomenclature of vegetation species was provided by Marhold, Hindák (1998). To evaluate changes in fundamental ecological factors by phytoindication, we used indirect information provided by Ellenberg indicator values (Ellenberg et al., 1991). To determine the main axes of variability of species data, we used indirect method for unimodal surgeries DCA ,Detrended correspodence analysis' in CANOCO (ter Braak, Šmilauer, 2002). Length gradient in the discriminatory analysis devoid of trends DCA was 6.775. indicating a need to use the unimodal method DCA. For the evaluation of species diversity, we used the Shannon index H> (Shannon, Weaver, 1963). The map output was processed in ArcMap 9.2.

According to the Slovak Catalogue of European Important Habitats, the 6210 semi-natural dry grasslands and scrubland facies on calcareous substartes (Festuco-Brometalia) and 6190 Rupicolous pannonic grasslands (StipoFestucetalia pallentis) belong to the European Important Habitats (EEC, 1992; Viceníková, Polák et al., 2003). When assigning taxa between invasive, expansive and synanthropy, we adhere to work Gojdičová et al. (2002) and actual field experience. Names of syntaxa is refers to the Janišová et al. (2007a), in the case of other types of vegetation by Mucina, Maglocký (1985).

\section{Results}

Repeated phytosociological research was carried out on selected stationary areas S 3, S 5, S 19 representing xerophilous grassland vegetation, and stationary areas S 9, S 10 representing phytocoenoses with dendroflora succession on former grassland. Rated stationary surfaces differ from each other by their natural conditions, vegetation, nature, position relative to 
roads and settlements and history of its creation and use. Currently, these areas are left to spontaneous development. For comparison purposes, they were characterized by two areas of forest ecosystems S 7 Fageto-Quercetum Zl. 59 and S 11 Fagetum typicum Zl. 59. For ecosystems of stationary area $S 19$ special protection regime has been developed, aimed at sustaining populations of protected plant species. The abandonment of agricultural use results in the development of successional phenomena.

All investigated communities in the period of 1985 and 2010 are described in the text. Indicator values according to Ellenberg and difference between the two monitored years of all records are shown in Table 1. Indices of species diversity of all records are shown in Table 2. DCA analysis with mark of individual floristic records are shown in Fig. 3. The tables and figure are listed in the Appendix.

T a ble 1. Indicator values according to ELLENBERG (1992) and difference between the two monitored years of all records.

\begin{tabular}{|c|c|c|c|c|c|c|c|c|}
\hline \multirow[b]{2}{*}{ Relevé } & \multirow[b]{2}{*}{ Year } & \multicolumn{7}{|c|}{ Ellenberg Indicator Values } \\
\hline & & Light & Teperature & Continentality & Moisture & $\begin{array}{c}\text { Soil } \\
\text { Reaction }\end{array}$ & Nutriens & $\begin{array}{c}\text { No. of all } \\
\text { species }\end{array}$ \\
\hline \multirow{3}{*}{9} & a - 1984 & 7.7 & 6.2 & 4.3 & 3.1 & 6.9 & 2.1 & 36 \\
\hline & b - 2010 & 7.4 & 5.8 & 4.6 & 3.3 & 7.3 & 2.9 & 43 \\
\hline & Difference & -0.3 & -0.4 & 0.3 & 0.2 & 0.4 & 0.8 & 7 \\
\hline \multirow{3}{*}{11} & $\mathrm{a}-1984$ & 4.9 & 5.2 & 3.7 & 5.3 & 6.4 & 5.8 & 60 \\
\hline & $\mathrm{b}-2010$ & 4.1 & 5.2 & 3.7 & 5.1 & 6.0 & 5.8 & 20 \\
\hline & Difference & -0.8 & 0.0 & 0.0 & -0.2 & -0.4 & 0.0 & -40 \\
\hline \multirow{3}{*}{19} & a - 1984 & 7.8 & 6.3 & 4.4 & 2.7 & 8.0 & 2.1 & 21 \\
\hline & $\mathrm{b}-2010$ & 7.6 & 6.2 & 4.5 & 3.2 & 7.5 & 2.8 & 51 \\
\hline & Difference & -0.2 & -0.1 & 0.1 & 0.5 & -0.5 & 0.7 & 30 \\
\hline \multirow{3}{*}{10} & $\mathrm{a}-1984$ & 7.1 & 5.8 & 3.8 & 4.2 & 5.4 & 3.2 & 38 \\
\hline & $\mathrm{b}-2010$ & 6.8 & 5.4 & 4.1 & 4.2 & 6.9 & 4.0 & 51 \\
\hline & Difference & -0.3 & -0.4 & 0.3 & 0.0 & 1.5 & 0.8 & 13 \\
\hline \multirow{3}{*}{7} & a - 1984 & 4.8 & 5.3 & 3.7 & 5.0 & 6.9 & 6.0 & 50 \\
\hline & b - 2010 & 3.5 & 5.3 & 2.8 & 5.2 & 6.7 & 7.0 & 7 \\
\hline & Difference & -1.3 & 0.0 & -0.9 & 0.2 & -0.2 & 1.0 & -43 \\
\hline \multirow{3}{*}{3} & a - 1984 & 6.4 & 6.0 & 4.0 & 3.8 & 7.4 & 3.7 & 61 \\
\hline & $b-2010$ & 6.9 & 6.0 & 4.0 & 3.7 & 7.4 & 3.6 & 81 \\
\hline & Difference & 0.5 & 0.0 & 0.2 & -0.1 & 0.0 & -0.1 & 20 \\
\hline \multirow{3}{*}{5} & a - 1984 & 7.6 & 6.2 & 4.7 & 3.0 & 7.6 & 2.5 & 50 \\
\hline & b - 2011 & 7.4 & 6.1 & 4.5 & 3.2 & 7.8 & 2.6 & 51 \\
\hline & Difference & -0.2 & -0.1 & -0.2 & 0.2 & 0.2 & 0.1 & 1 \\
\hline
\end{tabular}

$\mathrm{T}$ a b le 2. Indices of species diversity of all records in NP Slovenský kras.

\begin{tabular}{|l|c|c|c|c|c|c|c|c|c|c|c|c|c|c|}
\hline Relevé & 9a & 9b & $\mathbf{1 1 a}$ & $\mathbf{1 1 b}$ & $\mathbf{1 9 a}$ & $\mathbf{1 9 b}$ & $\mathbf{1 0 a}$ & $\mathbf{1 0 b}$ & $\mathbf{7 a}$ & $\mathbf{7 b}$ & $\mathbf{3 a}$ & $\mathbf{3 b}$ & $\mathbf{5 a}$ & $\mathbf{5 b}$ \\
\hline Richnes & 36 & 43 & 60 & 20 & 21 & 51 & 38 & 51 & 50 & 7 & 61 & 81 & 50 & 51 \\
\hline S-W index & 3.42 & 3.25 & 3.26 & 1.90 & 2.10 & 3.17 & 3.09 & 3.16 & 2.76 & 1.55 & 3.87 & 3.74 & 3.45 & 3.32 \\
\hline Evenness & 0.95 & 0.86 & 0.78 & 0.62 & 0.69 & 0.80 & 0.85 & 0.79 & 0.70 & 0.71 & 0.94 & 0.85 & 0.88 & 0.84 \\
\hline
\end{tabular}




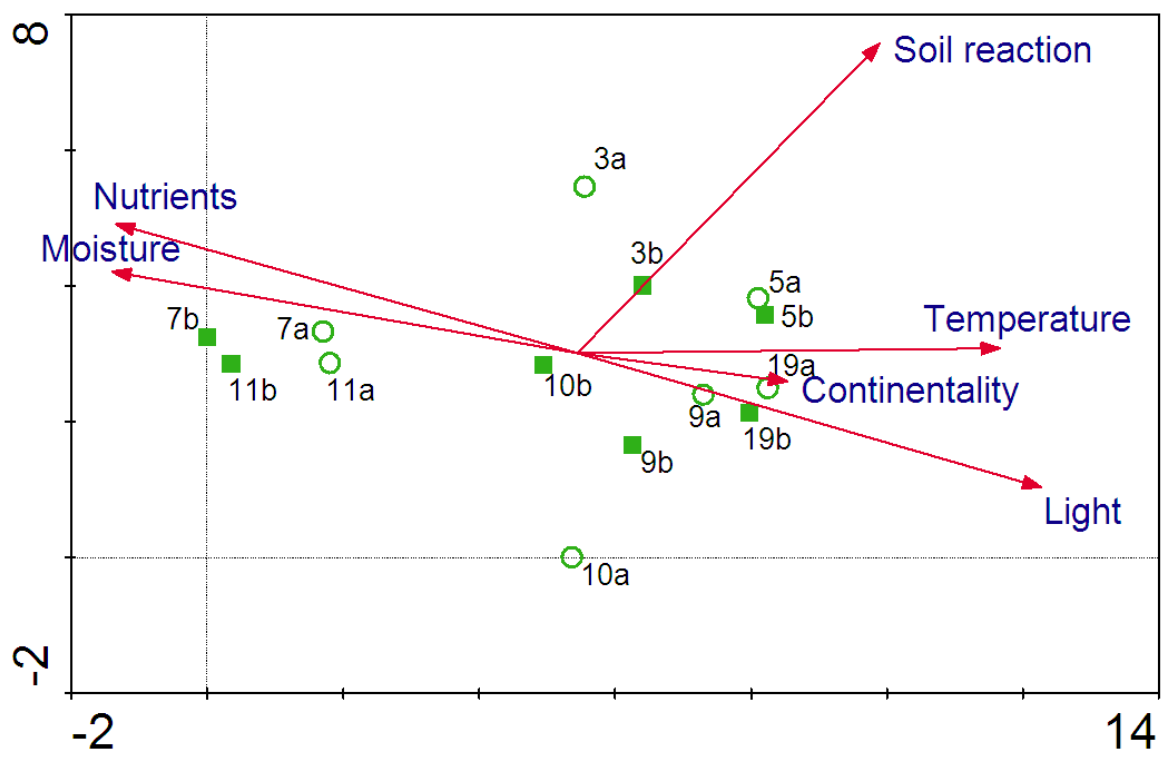

Fig. 3. DCA analysis with mark of individual floristic records (circles - first period, square - second period) with Ellenberg indicator values as supplementary variables (upper). The first two CA axes explain $62,7 \%$ of species and species-environment relation, respectively.

Phytocoenological characteristics of xerophilous grassland vegetation

Stationary area S3 - Železné vráta (Iron Gates), Plešivská Plateau, N 48³8`13,70“, E $20^{\circ} 23^{\prime} 34,40^{\prime \prime}$ about $10 \mathrm{~m}$ below the edge of the SW oriented steep limestone slope, $719 \mathrm{~m}$ a.s.l., slope 35 degrees, 35-40\% limestone rocks, E3 20\%, E2 30\%, E1 70\%, E0 30\%, 10×20 m, Uhliarová, 20.7.2010.

1985:

E3: Fraxinus ornus 2m, Cerasus mahaleb 1, Quercus cerris 1, Quercus pubescens agg. 1, Acer campestre +, Tilia cordata + , Sorbus aria + ,

E2: Berberis vulgaris 1, Cornus mas 1, Cerasus mahaleb 1, Rhamnus cathartica 1, Rosa canina agg. 1, Spiraea media 1, Fraxinus ornus + , Juniperus communis + , Prunus spinosa + , Populus tremula + , Ligustrum vulgare + , Ribes alpinum + , Euonymus verrucosus + , Quercus pubescens + , Cornus mas + , Viburnum lantana + , Sorbus aria + .

2010:

E3: Quercus pubescens (9 m), Fraxinus excelsior (4: 7,73 m), Fraxinus ornus, Cerasus mahaleb, Quercus cerris, Acer campestre, Carpinus betulus, Sorbus aria, Tilia cordata.

E2: Fraxinus excelsior $(0,3 \mathrm{~m})$, Juniperus communis $(0,6 \mathrm{~m})$, Ligustrum vulgare $(2,6 \mathrm{~m})$, Cornus mas (1,6 m), Berberis vulgaris (2,6, 0,6 m), Rosa canina (7: 1,31 m), Rhamnus catharticus, Spirea media (cca 50 jedincov: 0,5-0,75 m), Cerasus mahaleb, Prunus spinosa, Populus tremula, Ribes alpinum. 
E1: Sesleria heufleriana 4, Betonica officinalis $2 \mathrm{~m}$, Brachypodium pinnatum $2 \mathrm{~m}$, Crinitina linosyris 1, Chamaecytisus albus 1, Lembotropis nigricans 1, Festuca pallens 1, Galium mollugo agg. 1, Geranium sanguineum 1, Phleum phleoides 1, Salvia pratensis 1, Teucrium chamaedrys 1, Vicia cracca 1, Vincetoxicum hirundinaria 1, Achillea collina +, Alliaria petiolata + , Arenaria serpyllifolia +, Arrhenatherum elatius +, Asplenium ruta-muraria +, Asplenium trichomanes + , Lithospermum purpurocaeruleum + , Bupleurum longifolium + , Campanula bononiensis +, Campanula sibirica ssp. divergentiformis +, Campanula xylocarpa + , Carduus acanthoides + , Carex sp. + , Centaurea jacea ssp. oxylepis + , Echium vulgare +, Tithymalus epithymoides +, Fallopia dumetorum +, Fragaria moschata +, Galium verum +, Glechoma hirsuta +, Hylotelephium maximum +, Hypericum perforatum +, Jovibarba globifera ssp. hirta + , Laserpitium latifolium + , Melica ciliata + , Origanum vulgare + , Peucedanum cervaria + , Poa angustifolia + , Poa badensis + , Potentilla recta + , Primula veris + , Pseudolysimachion spicatum + , Silene latifolia + , Silene vulgaris + , Stachys recta + , Stellaria holostea + , Teucrium montanum + , Trifolium alpestre + , Verbascum lychnitis + , Veronica chamaedrys +, Viola tricolor +, Waldsteinia geoides +, Thymus pannonicus +, Aconitum anthora r, Erysimum odoratum r, Linaria pallidiflora r, Verbascum thapsus $\mathrm{r}$, Allium flavum $\mathrm{r}$

Synantropic species:

1985: Brachypodium pinnatum 2, Euphorbia cyparissias +, Fraxinus ornus 1

2010: Brachypodium pinnatum 1, Carduus acanthoides + , Tithymalus cyparissias + , Fraxinus ornus 2.

Stationary area S5 - Plešivec, the railway depot, Plešivská Plateau, N 48³3‘33,80“, E $20^{\circ} 25^{`} 04,40^{\prime \prime}$, exposure SW, $473 \mathrm{~m}$ a.s.1., 30-40 slope, limestone rocks 30-40\%, E2 35,\% E1 55\%, E0 25\%, 4x4m, Uhliarová, Hladká, 13.7.2011

E3: Cerasus mahaleb 1, Fraxinus ornus +, Viburnum lantana +,

E2: Berberis vulgaris 2a, Ligustrum vulgare $2 \mathrm{~m}$, Cornus mas 1 , Swida sanguinea 1 , Rosa canina agg. 1, Crataegus laevigata +, Viburnum lantana + ,

E1: Carex humilis 3, Potentilla arenaria 3, Festuca valesiaca 2a, Teucrium chamaedrys $2 \mathrm{~m}$, Viola hirta 1, Asperula cynanchica 1, Carduus collinus 1, Tithymalus cyparissias 1, Festuca pallens 1, Geranium sanguineum 1, Melica ciliata 1, Origanum vulgare 1, Poa badensis 1, Polygonatum odoratum 1, Stipa joannis 1, Stipa pulcherrima 1, Teucrium montanum 1, Thymus pannonicus 1, Thymus praecox 1, Arabis hirsuta + , Bothriochloa ischaemum + , Campanula sibirica ssp. divergentiformis + , Cleistogenes serotina + , Erysimum odoratum + , Galium album +, Galium glaucum +, Genista tinctoria +, Hypericum perforatum +, Koeleria macrantha +, Lactuca perennis + , Linaria pallidiflora + , Medicago falcata + , Medicago minima + , Pseudolysimachion spicatum + , Pulsatilla grandis + , Salvia pratensis + , Sesleria albicans + , Thalictrum minus + , Trifolium alpestre + , Veronica prostrata + , Vincetoxicum hirundinaria + , Adonis vernalis $\mathrm{r}$, Carlina vulgaris $\mathrm{r}$, Eryngium campestre $\mathrm{r}$, Jovibarba globifera ssp. hirta $\mathrm{r}$, Primula veris $\mathrm{r}$, Taraxacum erythrospermum $\mathrm{r}$.

Synantropic species:

1985: Tithymalus cyparissias +, Eryngium campestre +, Fraxinus ornus + 2010: Tithymalus cyparissias 1, Eryngium campestre +, Fraxinus ornus + 
Stationary area S19 - National Nature Reserve ,Domické škrapy - Domica Lapies)‘, Silická Plateau, N $48^{\circ} 28,49.15$, E $20^{\circ} 28^{\prime} 08.90^{\prime \prime}$, about $60 \mathrm{~m}$ from the educational panel No. 4, part of the moderate slope, exposure NNE, 390 m.a.s.l., slope 5, E2 5\%, E1 70\%, E0 65\%, limestone rocks on the surface of 30-35\%, $4 \times 4$ m, Uhliarová 19.7.2010, Šuvada, Uhliarová 22.5.2011.

1985:

E2: Rosa canina1, Juniperus communis 1, Crataegus curvisepala subsp. Domicensis 1 2010:

E2: Rosa canina agg. 1, Ligustrum vulgare 1, Prunus spinosa 1, Juniperus communis + E1: Dorycnium pentaphyllum agg. 3, Chamaecytisus albus 2b, Teucrium montanum 2b, Teucrium chamaedrys 2a, Festuca rupicola 2a, Seseli osseum 2a, Achillea nobilis 2m, Acosta rhenana $2 \mathrm{~m}$, Tithymalus cyparissias 2m, Agrimonia eupatoria 1, Asperula cynanchica 1, Carduus collinus 1, Carex humilis 1, Elytrigia intermedia 1, Eryngium campestre 1, Medicago lupulina 1, Potentilla arenaria 1, Prunella laciniata 1, Sanguisorba minor 1, Securigera varia 1, Verbascum lychnitis 1, Acinos arvensis +, Allium flavum +, Alyssum montanum ssp. brymii +, Anthericum ramosum +, Convolvulus arvensis +, Erigeron annuus +, Fragaria viridis +, Geranium columbinum +, Helianthemum nummularium +, Pilosella bauhinii +, Hypericum perforatum +, Juniperus communis + , Medicago minima + , Melica ciliata + , Plantago lanceolata + , Sedum sexangulare + , Senecio jacobaea + , Silene otites + , Stachys recta + , Taraxacum erythrospermum +, Tragopogon dubius + .

2011:

E2: Prunus spinosa 1, Rosa canina 1, Ligustrum vulgare 1, Pyrus pyraster +, Juniperus communis +.

E1: Carex humilis 3, Festuca rupicola 3, Potentilla arenaria 3, Teucrium chamaedrys 3, Chamaecytisus albus 2b, Arrhenatherum elatius 2m, Elytrigia intermedia 2m, Eryngium campestre $2 \mathrm{~m}$, Achillea nobilis 1, Dorycnium pentaphyllum agg. 1, Tithymalus cyparissias 1, Geranium columbinum 1, Koeleria macrantha 1, Securigera varia 1, Seseli osseum 1, Teucrium montanum 1, Achillea pannonica +, Acinos arvensis + , Agrimonia eupatoria + , Allium flavum + , Alyssum montanum ssp. brymii + , Anthericum ramosum + , Anthyllis vulneraria + , Asperula cynanchica + , Bothriochloa ischaemum + , Acosta rhenana + , Echium vulgare + , Stenactis annua + , Fallopia dumetorum +, Festuca valesiaca + , Helianthemum nummularium + , Pilosella bauhinii + , Hypericum perforatum + , Ligustrum vulgare + , Medicago lupulina + , Medicago minima + , Melica ciliata + , Phelipanche arenaria + , Plantago lanceolata + , Poa badensis + , Potentilla erecta + , Ranunculus bulbosus + , Rosa canina agg. +, Sanguisorba minor + , Scabiosa ochroleuca + , Sedum sexangulare + , Stachys recta + , Taraxacum erythrospermum + , Thymus praecox + , Tragopogon dubius + , Verbascum lychnitis + .

Synantropic species: Tithymalus cyparissias 2, Eryngium campestre 2, Cirsium arvense 2, Convolvulus arvensis + , Stenactis annua + .

Phytocoenoses with dendroflora succession on former grasslands

Stationary area S9 - Ostré vŕšky, Plešivská Plateau, N 48³7`23,20“, E 20²4`23,20“, NNE dimension sharp top $(775 \mathrm{~m})$, about $100 \mathrm{~m}$ above the bottom of a hole, SEE exposure $\left(120^{\circ}\right)$, 
650 m a.s.l., slope $25^{\circ}$, E2 1\%, E1 70\%, E0 65\%, Uhliarová, 19.7.2010.

1985:

E3: Carpinus betulus 1, Cerasus avium +.

E2: Berberis vulgaris 1, Corylus avellana 1, Crataegus laevigata +, Euonymus verrucosus +, Ribes uva-crispa +, Juniperus communis +, Pyrus communis +, Rosa sp. +, Viburnum lantana + E1: Brachypodium pinnatum 3, Achillea pannonica 2m, Calamagrostis epigejos 2m, Agrostis capillaris 1, Briza media 1, Cruciata glabra 1, Festuca valesiaca 1, Fragaria viridis 1, Galium verum 1, Geranium columbinum 1, Geranium sanguineum 1, Helianthemum grandiflorum ssp. obscurum 1, Phleum phleoides 1, Salvia pratensis 1, Securigera varia 1, Teucrium chamaedrys 1 , Allium senescens + , Betonica officinalis + , Bupleurum falcatum + , Carex caryophyllea + , Chamaecytisus albus +, Erysimum odoratum +, Euonymus europaeus + , Tithymalus epithymoides+, Festuca rupicola +, Galium album +, Melica ciliata +, Origanum vulgare + , Poa angustifolia + , Polygala comosa + , Populus tremula + , Potentilla recta + , Pseudolysimachion spicatum + , Sedum acre + , Teucrium montanum + , Thymus pannonicus + , Trifolium campestre + , Verbascum lychnitis + , Viola hirta + , Juniperus communis + ,

Carlina acaulis r, Dianthus pontederae r, Tithymalus cyparissias r, Knautia arvensis $\mathrm{r}$.

Synantropic species: Calamagrostis epigejos +, Tithymalus cyparissias +, Cirsium arvense +, Cirsium vulgare+.

2010:

E3: Betula pendula (19,5 m), Picea abies (11,3 m), Pinus sylvestris (9,6 m), Pyrus pyraster $(6 \mathrm{~m})$. E2: Juniperus communis (19: 1,74 m), Prunus spinosa (10: 0,55 m), Berberis vulgaris $(10: 1,18$ m), Betula pendula (11: 0,5 m), Quercus sp., Ligustrum vulgare (10: 2,16 m), Populus tremula (15: 0,5 m), Viburnum lantana, Cornus mas, Rosa canina, Rhamnus catharticus E1: Euonymus europaeus, Populus tremula

Synantropic species: Brachypodium pinnatum 3, Calamagrostis epigejos 2, Tithymalus cyparissias $\mathrm{r}$

Stationary area S10 - Ostré vŕšky, Plešivská Plateau, N 48³7`8,20“, E 20²4‘18,70“, NW oriented gentle slope near the adjacent dirt road $666 \mathrm{~m}$ a.s.l., slope $5^{\circ}$, E2 55\%, E1 15\%, E0 30\%, limestone rocks on the surface cover $30 \%$, area of the $4 \times 4 \mathrm{~m}$, Uhliarová, 19.7.2010. 1985:

E2: Juniperus communis 4, Acer campestre 1, Pinus sylvestris 1, Carpinus betulus +, Fagus sylvatica +, Ligustrum vulgare +, Lonicera xylosteum +, Picea abies +, Quercus petraea +, Rhamnus catharticus r, Corylus avellana 1 ,

2010:

E3: Acer campestre (6,3 m), Pinus sylvestris $(4,9 \mathrm{~m})$

E2: Juniperus communis (32: 2,78 m), Pinus sylvesris (20: 1,47 m), Quercus petrea, Rosa canina (3: 3,5 m), Picea abies (7: 0,56 m), Berberis vulgaris, Rhamnus cathartica, Carpinus betulus, Lonicera xylosteum, Ligustrum vulgare, Fagus sylvatica, Corylus avellana

E1: Brachypodium pinnatum 3, Agrostis capillaris 2a, Festuca rupicola 2m, Fragaria vesca 2m, Leontodon hispidus 2m, Poa compressa $2 \mathrm{~m}$, Poa pratensis agg. $2 \mathrm{~m}$, Achillea pannonica 1, Arrhenatherum elatius 1, Cruciata glabra 1, Galium verum 1, Helianthemum grandiflorum ssp. obscurum 1, Juniperus communis 1, Pimpinella saxifraga agg. 1, Plantago lanceolata 1, Rubus 
idaeus 1, Teucrium chamaedrys 1, Briza media + , Carex montana + , Cirsium arvense + , Dactylis glomerata + , Digitalis grandiflora + , Elytrigia repens + , Tithymalus cyparissias,+ Galium mollugo agg. +, Gentianopsis ciliata +, Hypericum perforatum +, Knautia arvensis + , Lotus corniculatus + , Plantago media + , Potentilla heptaphylla- + , Cerasus avium + , Ranunculus acris +, Salvia pratensis +, Thymus pulegioides +, Urtica dioica +, Veronica chamaedrys +, Viola hirta +, Juniperus communis +, Cerasus avium + Carlina acaulis r, Rosa canina agg. r, Stellaria graminea $\mathrm{r}$.

Synantropic species:

1985: Carduus nutans +

2010: Brachypodium pinnatum 3, Urtica dioica +, Cirsium arvense +, Elytrigia repens +

\section{Discussion}

Research of vegetation succession in the period 1985-2010 showed that the further development of grasslands, as well as drought-tolerant xerothermic plant communities in the karst area of Plešivská Plateau (Slovak Karst), is more influenced by progressive overgrowing of trees as well as occasional variations of temperature and precipitation. It can be predicted that in the longer term, today's herbal unused community will be replaced by forest communities that represent the climax stage of development of forest ecosystems.

Research in forest ecosystems of Slovak Karst confirmed the existence of stabilizing processes minimizing and counteracting the effects of climatic events, reinforcing community resilience. In agreement with some authors (Merlin et al., 2015), we can conclude that the stabilizing processes potentially contributing to this include attenuation compensation of the adult mortality caused by the event, due to site quality, variability to tolerance, phenotypic variability, plasticity at population level and the facilitating interactions. Reduced density in forest ecosystem affected by climatic conditions (winter, insect) gives rise to a large number of gap openings and new conditions favourable for seedling establishment or dispersed tree composition.

According to many authors, natural forests with complexity and integrity are able to withstand various stresses (Dhôte, 1994; Holling, 2001; Smith, 2001; Bréda et al., 2006; Weber et al., 2007; Galiano et al., 2010; Lloret et al., 2011, 2012; Cheaib et al., 2012; Lange et al., 2012; Michelot et al., 2012; Cavin et al., 2013; Vilá-Cabrera, 2013; Morán-Lopéz et al., 2014; Lawson et al., 2015; Merlin et al., 2015; Pötzelsberger et al., 2015) and they are not severely prone to negative functional climate change effects, and therefore, key forest functions can be maintained.

Dynamics of succession in non-forest vegetation on extreme sites of lapiés field

Potential vegetation according to Michalko et al. (1986) in stationary area S 3 (737 m, situated just below the edge of the karst plateau), is xerothermophilous submediterranian oak forests (Quercion pubescenti-petraeae pp) and rocky steppes (Seslerio-Festucion glaucae pp, Asplenia-Festucion glaucae). The communities belong to the European Important Habitat 6210 Semi-natural dry grassland and scrubland facies on calcareous substrates (Festuco-Bro- 
metalia). At the time of establishment of the stationary areas in 1983, a mosaic of lower loosening stands Corneto-Quercetum, rock vegetation and grassland vegetation of association Seslerietum heuflerianae and fringe communities of association Geranio-Trifolietum alpestris (Háberová, 1988; Vološčuk, 1988a) were recorded. After completion of the research, the surface was left to spontaneous development, when the area had a slightly increased diversity of grassland vegetation (68/56 vascular plant species) and smaller differences by protected and endangered plant species (Háberová, Karasová, 2002).

Higher species diversity of grassland vegetation (82/56 species) compared to 1983 , and the differences in coverage and composition of certain herbs, have also been observed with repeated phytocoenological and dendrological ratings of the stationary area in 2010 . The recorded changes have not yet caused change of grassland vegetation, which continues to meet the criteria for inclusion in association with Seslerietum heuflerianae. The observed differences in species composition of grassland vegetation are likely to be differences in climatic conditions in the evaluated years and slowly ongoing secondary succession raised by the richer representation of fringe species (Lembotropis nigricans, Geranium sanguineum, Betonica officinalis, Vincetoxicum hirundinaria), increased coverage of some trees (Fraxinus ornus, Quercus pubescens) and increasing overall species diversity (19/13 species) compared to 1983 . They totally cover an area of trees (50\%), which in comparison with 1983 is almost unchanged, suggesting that the vegetation areas close to its climax stage. On a stationary surface, we have not identified the spread of invasive or expansive plant species. Of other synantropic species, little coverage occurred in area with only individual pasture weeds Carduus acanthoides, Tithymalus cyparissias, expansive overgrown pasture species Brachypodium pinnatum and manna ash Fraxinus ornus, considered agriophyt of Central Europe (Lohmeyer, Sukopp, 1992). According to evaluation of Ellenberg ecological indexes (Table 1), there are no significant differentiation in vegetation succession between 1985 and 2010.

Stationary area S $\mathbf{5}$ is located in the southern part of Plešivská Plateau on karstic steep slope above the railway depot in Plešivec, which is considered the warmest area of the Slovak Karst. According to Michalko et al. (1986), the potential vegetation zone formed by xerothermophillous submediterranien oak forests (Quercion pubescenti-petraeae $\mathrm{pp}$ ) and rocky steppes (Seslerio-Festucion glaucae pp, Asplenio-Festucion glaucae). During the period of establishment, the stationary areas dominated on a slope, marked by the effects of soil erosion in the past, rocky steppe communities of association Poo-badensis-Caricetum humilis stipetosum and lower loosening stands Corneto-Quercetum. In the rocky steppe growths, significant populations of several endangered and protected species of plants were recorded and as a result the site has been designed for spontaneous development without afforestation. This led in subsequent years to intense proliferation of shrubs and spread enclaves of thermophilous oaks. Floristic area has been repeatedly evaluated in 2002 (Háberová, Karasová, 2002), which the authors recorded compared to 1983, and observed an increased diversity of vascular plants (65/46 species) and the retreat of several pioneer species, heliophyts and some protected plant species (Iris aphylla subsp. hungarica, Jurinea mollis subsp. macrocalathia, Onosma visianii, Pulsatilla grandis, Linaria pallidiflora).

With repeated phytocoenological and dendrological evaluation of areas in 2010, an increased diversity of steppe vegetation (56/46 species) due to its enrichment in tree species, 
fringe and forest species (Geranium sanguineum, Polygonatum odoratum, Fraxinus ornus, Swida sanguinea, Ligustrum vulgare, Viburnum lantana, etc.) and some herbaceous specieswas also observed. Further changes in vegetation were changes of quantitative representation of herbaceous species, in particular to increase coverage of cushion and tuft-growing species Potentilla arenaria, Festuca valesiaca and a coverage reduction of some heliophilous taxa (Festuca pallens, Poa badensis, Stipa pulcherrima, Rhodax canus). Despite these changes, the vegetation preserved the specific nature character of association Poo-badensis-Caricetum humilis, which was in recent years was also confirmed by Dúbravková-Michálková et al. (2008). Signs of continued secondary succession in rocky steppe vegetation is also higher coverage of some bushes (Berberis vulgaris, Ligustrum vulgare, Cornus mas) and increasing overall diversity of tree species (11/4 species), like in the previous case. An overview of recorded species and their characteristics are presented in the table. Currently wood covers $45 \%$ of stationary surface. Despite the nearby fields and the railway station in the stand, we neither recorded the spread of invasive plant species nor its expansive spread. The coverage was slightly increased only for species Tithymalus cyparissias remaining in the stand long past the intensive grazing in locations and woody agriofyt Fraxinus ornus.

According to evaluation of Ellenberg ecological indexes, there is no significant differentiation in vegetation succession between 1985 and 2010.

Stationary area S 19 Domické lapiés field is situated in the southwestern part of the Silica Plateau above the Domica cave on slightly sloping hillside covered with lapiés field. The area is part of National Nature Reserve Domické škrapy. According to Michalko et al. (1986), the potential vegetation represent cerium-oak forests (Quercetum petraeae-cerris sl). At the time of establishment of the stationary area, in 1985, it covered pastures thermophilic assotiation Poo-badensis-Caricetum humilis incurred due to natural conditions and long-term grazing, which was concluded before the foundation of the stationary surfaces (Vološčuk, 1993). The absence of grazing promotes the spread of thermophilic shrubs that threatens the existence of non-forest vegetation, which bears the appearance of a number of protected and endangered plant species. Therefore, Administration of NP Slovak Karst carries out occasional removal of shrubs, especially in the lower-lying parts of the slope off the stationary surface where a rare population of thermophilic species Echium russicum is also found.

Evaluation results of the stand showed that it took place, compared to 1985, to exchange the landmarks of grass vegetation, as well as to a change in the vegetation communities indigenous to the association Festuco rupicolae-Caricetum humilis. The presence of this association for the station confirmed the findings of Dúbravková-Michálková et al. (2008), by which the current dominant vegetation, Festuca valesiaca and F. rupicola, bound by their occurrence in deeper and mor humid soils was originally prevailing over Carex humilis and Poa badensis. The observed changes in species composition of grassland vegetation is likely increased the formation of soil from accumulating litter and intensive accumulation, supported significantly by less prone of area compared to the steep limestone tent. Its role in changing plant community could play well as changes in temperature and humidity conditions as a consequence of a larger habitat shading shrubs. Besides changes of grassland vegetation on a stationary surface, we saw a minor increase in species diversity of plants $(6 / 3$ species), the total cover (12\%) but it is a result of the occasional removal of bushes clearly 
smaller than the area left stationary for spontaneous development, in addition to threatening the species xerotherm steppe vegetation and the spread of invasive taxon Stenactis annua, whose resources are likely to be close to the roads and fields and expanding populations of grazing and arable weed Eryngium campestre, Tithymalus cyparissias and Cirsium arvense.

According to evaluation of Ellenberg ecological indexes (Table 1), there are significant differentiation in vegetation succession between 1985 and 2010 in terms of nutrients (defferentiation 0.7$)$, moisture (0.5) and soil reaction $(-0.5)$.

\section{Dynamics of succession in non-forest vegetation on deeper soils}

Stationary area S 9 is a bord of pit in the western part of Plešivská Plateau under the skin Ostré vŕšky $(775 \mathrm{~m})$. Potential vegetation according to Michalko et al. (1986) is oak-hornbeam-birch forest of the Carpathians (Tilio-Carpinenion betuli), on the rocky slopes of karst pits, and oak thermophilic steppe. In 1985, they formed a stationary surface thermophilic vegetation and pastures ass. including Alysso heterophylli-Festucetum valesiacae with the occurrence of protected species Stipa pulcherrima and Pulsatilla grandis (Háberová, 1988, Vološčuk, 1988a). After completion of the research, the stationary space was left to spontaneous development without afforestation and floristic re-evaluated in 2002 (Háberová, Karasová, 2002), which the authors recorded in the stand spread Brachypodium pinnatum. By now, this species in the stand prevails. Based on the results of the phytosociological assessment in 2010, the grassland vegetation of the stationary surface is a more advanced stage of secondary succession, which indicates the number of border species (Lembotropis nigricans, Origanum vulgare, Betonica officinalis) and high dominance of expansive grasses (Brachypodium pinnatum, Calamagrostis epigejos), which in the stand almost completely suppressed populations of most native species. Development of vegetation on the surface is accompanied by an increase in species diversity of trees, which reaches the coverage is currently $40 \%$. A different course of secondary succession on the slopes of karst pits in comparison with steep karst slopes lower elevations, accompanied by expansive spread of grasses, is conditioned by the presence of deeper and wetter soils.

According to evaluation of Ellenberg ecological indexes, there are no significant differentiation in vegetation succession between 1985 and 2010.

Stationary area S $\mathbf{1 0}$ is located in the western slope of the Plešivská Plateau, in shallow karst pits (sinkholes) under the skin Ostré vŕšky (775 m). According to Michalko et al. (1986), the vegetation belongs to oak-hornbeam forests of the Carpathians (Tilio-Carpinenion betuli). At the time of establishment of the stationary plots, the area was covered by association Campanullo rotundifolii-Dianthetum deltoidis from the union Violion caninae (Háberová et al. 1987; Vološčuk, 1988a), indicating a significant acidification of the upper layers of the soil. After allowing of area for the spontaneous evolution, relatively rapidly overgrowth of grassland vegetation by trees occurred, because in less than 20 years, this created an almost complete canopy of pioneer tree species (Juniperus communis, Betula pendula, Picea abies and Pinus sylvestris) that the shadowing stand completely changed its composition (Háberová, Karasová, 2002; Stančič, 2010).

The high degree of overgrown stationary surface was also confirmed by repeated phytosociological and dendrological research in 2010, when the coverage of trees was estimated at 
$60 \%$. Among the trees the taxon Juniperus communis prevailed, in which individuals totalled more than $2.5 \mathrm{~m}$. The rest consisted mainly of deciduous species, indicating the evolution of vegetation towards the original oak-hornbeam forests. In the herb layer Brachypodium pinnatum and some meadow grass prevailed and we recorded it in a more synanthropy species (Cirsium arvense, Tithymalus cyparissias, Urtica dioica), but given the large shading trees it had only little coverage.

\section{Dynamics of succession changes in forest habitats}

Stationary area S 7 in the forest ecosystem underwent a significant change between 1985 and 2010 by tree composition and number of tree species.

A significantly increased composition of the strong C strategist Quercus petraea was visible at the expense of C-R strategist Carpinus betulus. Fraxinus excelsior is C-R-S strategist with the ability to endure stress and after disturbances quickly occupy the gaps. The total involvement of crowns fell in that period from 95 to $85 \%$. This is reflected in the change in total volume of timber, circular base and the number of trees. In layer E2, the share of Carpinus betulus in both periods was $20 \%$, indicating its ability to assert itself against its competitors oak characteristics, i.e. a longer period of vegetative growth in youth. Natural regeneration of oak and beech partly in the layer to $50 \mathrm{~cm}$ is favourable. The best naturally restored C-R-S strategist is Fraxinus excelsior. Natural regeneration of oak, beech and ash are undermined by herbivorous animals. This causes disruption of the natural reproductive cycle of the forest ecosystem. Analysis of organic figures shows that in the time period 1985-2010 in the soil (rendzina) other than the exception of nitrogen, no significant change was observed in the average econumbers of light, humidity and soil acidity. If the nitrogen content in soil led to a small increase in interdependence in the monitored period, it reflected the movement of the species with the middle rich soils of the types to rich soils. The relationship between soil reaction and nitrogen, and between temperature and nitrogen, can be explained by seasonal dynamics of increasing species' abundance because of intensive humus and nitrogen in the soil and also changes in the dynamics of tree species in the past 25 years. Similarity index (Jaccard index 100\%, Sörensen index 100\%) of plant communities of the two periods shows the relative stability of the structure of plant communities. It also confirms the high index of dominance in the period (Cn 76.10 and $75.85 \%)$.

Stationary area $\mathbf{S} 11$ beech forest ecosystem Fagetum typicum Zl. 59 of mesotrophic ecological habitats and beech vegetation belts (Zlatník, 1959) is interesting in terms of the occurrence of artificial spruce monocultures (Picea abies), which, in 2004, was affected by windbreaks. It grows at an altitude of 620 m.a.s.l, with a slope of $3-5^{\circ}$. Soil is rubefic rendzinas.

Beech forest ecosystems in Plešivská Plateau occur generally in inverse areas of karst sinkholes or depressions with flat earth surface. The tree species composition is often dominated by spruce, whose authenticity in Plešivská Plateau is not yet clear with a scientific explanation. During the spread of vegetation in postglacial time, the spruce in the Slovak Karst was probably native trees (Ložek, 1973).

Forest ecosystem of stationary area in the period 1985-2010 was constantly disrupted by the wind and also intentional harvesting of spruce. The reduction of canopy crowns speed- 
ed up litter mineralization and the onset nitrophilous vegetation. Opening the side wall of spruce forests has enabled wind contradicted trees. The gaps without trees penetrated nitrophilous types of forest vegetation. In natural regeneration, C-R-S strategists Acer pseudoplatanus and Acer platanoides (67,000 units per ha), but also ash and beech, dominated. In a layer over $50 \mathrm{~cm}$, in addition to maple, a competitive C strategist Fagus sylvatica is also seen. Reducing the high number of individuals in a layer of $50 \mathrm{~cm}$ is a major factor in attracting herbivorous animals.

Tree species in 1985 was spruce $100 \%$, present as beech, hornbeam, oak and aspen. In the layer of $50 \mathrm{~cm}$ positive values of coverage were observed for sycamore maple, Norway maple, hornbeam and beech. The coverage of herbs layer was 60-90\%. Dominant and subdominant beech herbs also occurred.

After the wind calamity in 2004, the resulting area without trees started secondary succession herbs with dominant species Brachypodium pinnatum, Urtica dioica and Rubus hirtus. The natural regeneration of up to $50 \mathrm{~cm}$ occurred in the woods: beech $55 \%$, hornbeam $20 \%$, maple $5 \%$, spruce + .

A comparison figures for organic plant community from 1985 and 2010 shows a slight increase in the index of nitrogen in the soil, which is associated with bloom vegetation and improving humification processes of cambisols. The value of ecological indexes in the compared periods have quite a standoff. Stationary surface is suitable for further long-term follow-up of successional processes on the forests after the wind calamity.

\section{Conclusion}

Flora and vegetation of the Slovak Karst is extremely rich in species, which is conditioned to limestone bedrock and mainly overlap between floristic elements of Pannonian and Carpathian phyto-geographical regions (Rozložník, Karasová, 1994; Šuvada, 2008). A high biodiversity area is largely involved in the non-forest habitats, and its area constantly shrinking due to changes in land use. Secondary succession, conditioned to management changes, on the non-forest habitats is influenced by many factors and in addition, the spread of species is accompanied by a change in the species composition of the grassland vegetation.

Xerophylous and mesic grassland vegetation of most non-forest stationary areas of the Slovak Karst is no longer of economic use, leading to their overgrowing trees. Speed in growth and the current coverage of trees is mainly dependent on habitat conditions, primarily the depth of the soil and terrain inclination and since the end of economic exploitation stand. Stationary areas left to spontaneous development have now overgrown trees to $40-60 \%$ of the area, with the exception of locations with extremely shallow and undeveloped land where the coverage is greatly reduced due to unsuitable conditions for their growth.

On the steep limestone slopes, the ongoing secondary succession of trees and grassland vegetation are relatively slow and mainly influenced by soil depth and speed of its work from the accumulated litter. In the case of grassland vegetation in the first years or decades mainly, it reflected changes in the quantitative representation of herbaceous species, but overall species composition of present vegetation in relation to the vegetation communities saw no sub- 
stantial change. Therefore, to maintain the populations of rare and endangered plant species in karst hillsides, occasional removal of bushes is usually enough, which results in addition to greater use of daylight crop smaller accumulation of litter, which helps block the succession and the accompanying vegetation changes.

On flat terrain of lapiés fields, faster production and accumulation of soil similar to steep karst slopes occurred after the cessation of management for, and it caused less soil to flush out the few pitched field torrential rains, which characteristic of the Slovak Karst. The increased accumulation of soil is accompanied by rapid circulation of plants and pronounced changes in species composition of the grassland vegetation, which may lead to a change of its plant community. For these reasons, rare plant communities and populations of protected plant species should be maintained at these stations and it is essential to ensure the removal of trees at least minimal management stands only in the form of cutting or grazing (Cavin et al., 2013).

On the deeper, moister soils of plateaux, ongoing succession of species compared to lower elevations of lapiés fields is much faster. Creating a continuous canopy of trees on unused grasslands may take a longer time of nearly 20 years. Succession plants in these conditions, accompanied by overgrowing of grassland vegetation Brachypodium pinnatum and Calamagrostis epigejos that displace native species of vegetation, thus completely change its species composition. Restoring the original species composition of vegetation, overgrown by expansive grasses, is very difficult, slow, scarce and in relation to the rare taxa often unsuccessful (Ružičková et al., 2001). Therefore, to maintain species diversity of grassland vegetation to plateaux is necessary ensure at least the minimum economic use of vegetation. The appropriateness of a management for the preservation of rare plant communities and protected plant species is best tested in permanent research plots, established directly in the Slovak Karst National Park for this purpose.

The development of vegetation in most of the examined stationary surfaces was accompanied by an increase in total species diversity of vascular plants, increasing species diversity and species coverage, changes in quantitative representation of herbaceous species and some stationary surfaces and changing plant community. On the evaluated surfaces, we did not observe a significant spread of invasive species, probably due to extreme site conditions and/ or their localization outside the busy roads and waterways. Invasive plant (Stenactis annua) is only located at the most visited locations in stationary S 19 Domické lapiés fields. Of other synanthropic species, higher coverage of some locations, including warmer locations, occurred for pasture weeds Tithymalus cyparissias and Eryngium campestre and trees Fraxinus ornus considered as agriofyt of Central Europe.

The current state of ecological processes in the stationary surfaces of forest ecosystems allows them to predict the direction of the development of ecologically stable natural forests in the next decades, only by ensuring the protection of ecosystems prior to human disturbance (Grimm, Wissel, 1997). State forestry and state conservancy administrations are two possibilities by leading the development of ecosystems in stationary surfaces for environmentally stable climatic communities by irreplaceable scientific research and educationalenvironmental functions, or ensuring their rational economic use for the purpose of gaining maximum benefit from forest ecosystem services. 


\section{Acknowledgements}

This work was funded by the Grant Agency VEGA No. 1/0172/12 and No. 1/0255/14. The authors also wish to thank Jan Kilík, Milan Hlivák, Robert Šuvada, Jozef Rogos and Drahomira Hladká for the field work and their support in collecting the data.

\section{References}

Anonymous (1992). Council Directive 92/43/EEC of 21 May 1992 on the conservation of natural habitats and of wild fauna and flora. The Council of the European Communities.

Braun-Blanquet, J. (1964). Pflanzensociologie. Grundzüge der vegetationskunde. Wien, New York: Springer Verlag.

Bréda, N., Huc, R., Granier, A. \& Dreyer E. (2006). Temperate forest trees and stands under severe drought: a review of ecophysiological responses, adaptions processess and long-term consequences. Ann. For. Sci., 63, 625-644. DOI: $10.1051 /$ forest:2006042.

Capra, F. (1997). The web of life: A new synthesis of mind and matter. London: Hammersmith.

Cavin, L., Mountford, E.P., Peterken, G.F. \& Jump A.S. (2013). Extreme drought alters competitive dominance within and between tree species in a mixed forest stand. Funct. Ecol., 27, 1424-1435. DOI: 10.1111/1365-2435.12126.

Cheaib, A., Badeau, V., Boe, J., Chuine, I., Delire, Ch., Dufrêne, E., François, Ch., Gritti, E.S., Legay, M., Pagé, Ch., Thuiller, W., Viovy, N. \& Leadley P. (2012). Climate change impacts on tree ranges: modelintercomparison facilitates understanding and quantification of uncertainty. Ecol. Lett., 15(6), 533-544. DOI: 10.1111/j.14610248.2012.01764.x.

Devillers, P. \& Devillers-Terschuren J. (1999). Palearctic habitats classification. Strasburg: Council of Europe.

Dhôte, J.F. (1994). Hypothesis about competition for light and water in even-aged common beech (Fagus sylvatica L.). For. Ecol. Manag., 69, 219-232. DOI: 10.1016/0378-1127(94)90231-3.

Dúbravková-Michálková, D., Janišová, M., Kolbek, J., Šuvada, R., Virók, V. \& Zaliberová M. (2008). Dry grasslands in the Slovenský kras Mts (Slovakia) and the Aggteleki-karszt Mts (Hungary) - a comparison of two classification approaches. Hacquetia, 7(2), 123-140. DOI: 10.2478/v10028-008-0007-2.

Ellenberg, H., Weber, H.E., Düll, R., Wirth, V., Werner, W. \& Paulisen D. (1991). Zeigerwerte von Pflanzen in Mitteleuropa. Scripta Geobot., 18, 1-248.

Galiano, L., Martínez-Vilalta, J. \& Lloret F. (2010). Drought-induced multifactor decline of scots pine in the Pyrenees and potential vegetation change by the expansion of co-occuring oak species. Ecosystems, 13, 978-991. DOI: $10.1007 / \mathrm{s} 10021-010-9368-8$.

Glenn-Lewin, D.C. \& van der Maarel E. (1992). Patterns and processes of vegetation dynamics. In D.C. GlennLewin, R.K. Peet \& T.T. Veblen (Eds.), Plant succession - theory and prediction (pp. 11-59). London: Chapman \& Hall.

Gojdičová, E., Cvachová, A. \& Karasová E. (2002). List of non-native, invasive and expansive plants in Slovakia 2 (in Slovak). Ochrana Prírody, 21, 39-58.

Grime, J.P. (2001). Plant strategies, vegetation processes, and ecosystem properties. Chichester: John Wiley \& Sons.

Grimm, V. \& Wissel Ch. (1997). Babel, or the ecological stability discussions: an inventory and analysis of terminology and a guide for avoiding confusion Oecologia, 109, 323-334. DOI: 10.1007/s004420050090.

Háberová, I. et al. (1987). Vegetácia krasových oblastí SSR z hladiska ochrany. Msc. Záverečná správa štátneho plánu základného výskumu VI-3-3/03 za 7. 5RP. Prírodovedecká fakulta UK v Bratisdlave, 178 pp.

Háberová, I. (1988). Flora and vegetation of Plešivská Plateau (in Slovak). Výskumné Práce z Ochrany Prírody, 6B, 5-96.

Háberová, I. \& Karasová E. (2002). Floristic changes of nonforexst vegetation in Plešivská Plateau (in Slovak). In R. Midriak (Ed.), Biosférické rezervácie na Slovensku IV (pp. 111-116). Zborník referátov. SNV MAB.

Hennekens, S.M. \& Schaminée J.H.J. (2001). TURBOVEG, a comprehensive data base management system for vegetation data. J. Veg. Sci., 12, 589-591. DOI: 10.2307/3237010.

Hill, M.O. \& Šmilauer P. (2005). TWINSPAN for Windows version 2.3. České Budějovice: Centre for Ecology \& Hydrology and University of South Bohemia, Huntingdon.

Holling, C.S. (2001). Understanding the complexity of economic, ecological, and social systems. Ecosystems, 4 , 390-405. DOI: 10.1007/s10021-001-0101-5.

Interpretation manual of European Union Habitats, (2003). European Commission DG Environment, Nature and Biodiversity, 127 pp. 
IPCC (2013). Summary for Policymakers. In T.F. Stocker, D. Quin, G.-K. Plattner, M. Tignor, S.K. Allen, J. Boschung, A. Nauels, Y. Xia, V. Bex \& P.M. Midgley, (Eds.), Climate change: The physical science basis. Contribution of Working Group 1 to the Fifth Assessment Report of the Intergovernmental Panel on Climate Change. Cambridge University Press, Vambridge, United Kingdom and New York, NY, USA.

IUSS Working Group WRB (2014). World Reference Base for Soil Resources 2014. International soil classification system for naming soils and creating legends for soil maps. World Soil Resources Reports No. 106. FAO, Rome.

Janišová, M., Hájková, P., Hegedüšová, K., Hrivnák, R., Kliment, J., Michálková, D., Ružičková, H., Řezníčková, M., Tichý, L., Škodová, I., Uhliarová, E., Ujházy, K. \& Zaliberová M. (2007a). Grassland and herb vegetation of Slovakia (in Slovak). Elektronický expertný systém na identifikáciu syntaxónov. Bratislava: Botanický ústav SAV.

Janišová, M., Hrivnák, R., Gömöry, D., Ujházy, K., Valachovič, M., Gömöryová, E., Hegedüšová, K. \& Škodová I. (2007b). Changes of understorey vegetation after Norway spruce colonization of an abandoned grassland. Ann. Bot. Fenn., 44, 256-266. http://www.annbot.net

Jörgensen, S.E. \& Svirezhev Y.M. (2004). Towards a thermodynamic theory for ecological systems. Oxford: Elsevier.

Kay, J.J. (2000). Ecosystems as self-organizing holarchic open systems: narratives and the second law of thermodynamics. In: S.E. Jorgenses \& F. Müller (Eds.), Handbook of ecosystem theories and management (pp. 135-160). CRC Press -Lewis Publishers.

Lange, B., Germann, P.F. \& Lüscher P. (2012). Greater abundance of Fagus sylvatica in coniferous flood protection forests due to climate change: impact of modified root densities on infiltration. European Journal of Forest Research, 132, 151-163. DOI: 10.1007/s10342-012-0664-z.

Lawson, C.R., Vindenes, Y., Bailey, L. \& van de Pol M. (2015). Environmental variation and population responses to global change. Ecol. Lett., 18(7), 724-736. DOI: 10.1111/ele.12437.

Lloret, F., Keeling, E.G. \& Sala A. (2011). Components of tree resilience: effects of successive low-growth episodes in old pondelosa pine forests. Oikos, 120, 1909-1920. DOI: 10.1111/j.1600-0706.2011.19372.x.

Lloret, F., Escudero, A., Iriondo, J.M., Martinez-Vilalta, J. \& Valladeres F. (2012). Extreme climatic events and vegetation: the role of stabilizing processes. Global Change Biology, 18, 797-805. DOI: 10.1111/j.13652486.2011.02624.

Lohmeyer, W. \& Sukopp H. (1992). Agriophyten in der vegetation Mitteleuropas. Schr.-Reihe Vegetationskunde 25. Bonn, Bad Godesberg.

Ložek, V. (1973). The nature in quaternary (in Czech). Praha: Academia.

Marhold, K. \& Hindák F. (Eds.) (1998). Checklist of non-vascular and vascularv plants of Slovakia (in Slovak). Bratislava: Veda.

Michalko, J., Berta, J. \& Magic D. (1986). Geobotanical map of ČSSR (in Slovak). Bratislava: Veda.

Michelot, A., Bréda, N., Damesin, C. \& Dufrene E. (2012). Differing growth responses to climatic variations and soil water deficit of Fagus sylvatica, Quercus petraea and Pinus sylvestris in a temperate forest. For. Ecol. Manag., 265, 161-171. DOI: 10.1016/j.foreco.2011.10.024.

Merlin, M., Perot, T., Perret, S., Korboulewsky, N. \& Vallet P. (2015). Effects of stand composition and tree size on resistance and resilience to drought in sessile oak and Scots pine. For. Ecol. Manag., 339, 22-33. DOI: 10.1016/j. foreco.2014.11.032.

Morán-Lopéz, T., Poyatos, R., Llorens, P. \& Sabaté S. (2014). Effects of past growth trends and current water use strategies on Scots pine and pubescent oak drought sensitivity. European Journal of Forest Research, 133, 369-382. DOI: $10.1007 / \mathrm{s} 10342-013-0768-0$.

Mucina, L. \& Maglocký Š. (Eds.) (1985). A list of vegetation units of Slovakia. Docum. Phytosociol. N.S., Camerino, 9, 175-220.

Odum, E.P. (1969). The strategy of ecosystem development. Science, 164, 262-270. DOI: 10.1126/science.164.3877.262

Pickett, S.T.A., Collins, S.L. \& Armesto J.J. (1987). Models, mechanisms and pathways of succession. Bot. Rev., 53, 335-371. DOI: 10.1007/BF02858321.

Pötzelsberger, E., Wolfslehner, B. \& Hasenaue H. (2015). Climate change impacts on key forest functions of the Vienna woods. European Journal of Forest Research, 134(3), 481-496. DOI: 10.1007/s10342-015-0866-2.

Rozložník, M. \& Karasová E. (Eds.) (1994). Slovak Karst Protected Landscape Area, Biosphere Reserve (in Slovak). Martin: Osveta.

Ružičková ,H., Halada, L., David, S., Gerhátová, K. (2001). Management of meadows in the Biosphere Reserve East Carpathians. II. Results after 6 years. Ekológia (Bratislava), 20(Suppl. 3), 76-87.

Sabo, P., Uhliarová, E. \& Turisová I. (2011). The concepts of ecological complexity and integrity and possibilities to their using in ecosystem research in Slovak Karst (in Slovak). In I. Vološčuk et al. (Eds.), Dynamika sukcesných 
procesov, štruktúry a ekologickej integrity ekosystémov Slovenského krasu (pp. 191-214). Banská Bystrica: Univerzita Mateja Bela.

Shannon, C.E. \& Weaver W. (1963). The mathematical theory of communication. University of Illionois Press.

Smith, M.D. (2011). An ecological perspective on extreme climatic events: a synthetic definition and framework to guide future research. J. Ecol., 99, 656-663. DOI: 10.1111/j.1365-2745.2011.01798.x.

SNC, Slovak National Committee for UNESCO MAB Programme (2011). Periodic review for Slovak Karst Biosphere Reserve. Manuscript. Bratislava: ÚKE SAV.

Stančič, Z. (2010). Marshland vegetation of the class Phragmito-Magnocaricetea in northwest Croatia (Krapina river valley). Biologia, Bratislava, 65(1), 39-53. DOI: 10.2478/s11756-009-0232-2.

Šilc, U. \& Čarni A. (2005). Changes in weed vegetation on extensively managed fields of central Slovenia between 1939 and 2002. Biologia, Bratislava, 60, 409-416.

Šuvada, R. (2008). List of endemic, protected and endangered high plants of Slovak flora (in Slovak). In R. Midriak \& L. Zaušková (Eds.), Biosférické rezervácie na Slovensku VII. (pp. 49-59).

Ter Braak, C.J.F. \& Šmilauer P. (2002). CANOCO reference manual and CanoDraw for Windows user's guide. Software for Canonical Community Ordination (version 4.5). Microcomputer Power, Ithaca.

Tichý, L. (2002). JUICE, software for vegetation classification. J. Veg. Sci., 13, 451-453. DOI: 10.1111/j.1654-1103.2002. tb02069.x.

Tichý, L. \& Holt J. (2006). JUICE program for management, analysis and classification of ecological data. Vegetation science group. Brno: Masaryk University.

Tichý, L. \& Chytrý M. (2006). Statistical determination of diagnostic species for site groups of unequal size. J. Veg. Sci., 17, 809-818. DOI: 10.1111/j.1654-1103.2006.tb02504.x

Van der Maarel, E. (1979). Transformation of cover-abundance values in phytosociology and its effects on community similarity. Vegetatio, 39, 97-114. DOI: 10.1007/BF00052021

Viceníková, A. \& Polák P. (Eds.) (2003). Európsky významné biotopy na Slovensku. Banská Bystrica: ŠOPSR.

Vilá-Cabrera, A., Martinez-Vilalta, J., Galiano, L. \& Retana J. (2013). Patterns of forest decline and regeneration across scots pine populations. Ecosystems, 16, 323-335. DOI: 10.1007/s10021-012-9615-2.

Vološčuk, I. (1988a). The characteristics of stationary plots of Plešivská Plateau (in Slovak). Výskumné Práce $z$ Ochrany Prírody, 6B, 99-142.

Vološčuk, I. (1988b). Analyse of dendroflora in stationary plots (in Slovak). Výskumné Práce z Ochrany Prírody, 6B, 325-353.

Vološčuk, I. (1993). Biotops, phytocoenoses, structure and productivity of forest canopy in stationary areas of Nature Protection in Slovak Karst (in Slovak). Ochrana Prírody, 12, 57-103.

Vološčuk, I., Uhliarová, E., Midriak, R., Sabo, P., Hladká, D., Lepeška, T., Korónyi, S., Zaušková, L., Bošela, M., Šebeň, V., Šuvada, R. \& Rogos J. (2011). The dynamics of succession processes, structure and ecological integrity of Slovak Karst Ecosystems (in Slovak). Banská Bystrica: Univerzita Mateja Bela.

Weber, P., Bugmann, H. \& Rigling A. (2007). Radial growth responses to drought of Pinus sylvestris and Quercus pubescens in an inner-Alpine dry valley. J. Veg. Sci., 18, 777-792. DOI: 10.1111/j.1654-1103.2007.tb02594.x.

Wu, J. \& Hobbs R. (2002). Key issues and research priorities in landscape ecology: An idiosyncratic synthesis. Landsc. Ecol., 17, 355-365. DOI: 10.1023/A:1020561630963.

Würtz, P. \& Anilla A. (2010). Ecological succession as an energy dispersal process. Biosystems, 100(1), 70-78. DOI: 10.1016/j.biosystems.2010.01.004.

Yamamoto, S.I. (2000). Forest gap dynamics and tree regeneration. Journal of Forest Research, 5, 223-229. DOI: 10.1007/BF02767114.

Zhang, H. \& Wu J. (2002). A statistical thermodynamic model of the organizational order of vegetation. Ecol. Model., 153, 69-80. DOI: 10.1016/S0304-3800(01)00502-6.

Zlatník, A. (1959). Survey of the Slovak forest according to group of forest types (in Czech). Brno: Lesnícka fakulta VŠZ. 